REVISTA DE DERECHO UNED, NÚM. 12, 2013

\title{
EL ESTATUTO JURÍDICO DE LAS FUERZAS ARMADAS
}

\author{
LEGAL STATUS OF THE ARMED FORCES
}

JoAQuín LÓPEZ DE GEA

Licenciado en Derecho. Diploma en Estudios Avanzados en Derecho

Constitucional. UNED. Teniente de Infantería en la Reserva

\begin{abstract}
The 8th article of the Spanish Constitution lists the missions of the Armed Forces. These Armed Forces are an Administration with special features relative to personnel legal regulations, operative procedures, values, targets and purposes. This article tries to go through the Army legal regulations, underlining the analysis of its nature and missions in order to update the current legal literature. To sum it up, a big addition of missions to the "status quo" of the Armed Forces recently happened, brought as a result of the international integration of Spain; and, secondly, the Armed Forces may be considered as an «institutional Administration».
\end{abstract}

Resumen: El artículo 8 de la Constitución Española enumera las misiones de las Fuerzas Armadas. Éstas constituyen una Administración con singularidades en materia de régimen de personal, procedimientos operativos, valores, objetivos y funciones. En el presente artículo revisamos, con ánimo de actualizar la literatura jurídica existente, el régimen jurídico de las Fuerzas Armadas, poniendo especial énfasis en el análisis de su naturaleza y de sus misiones. De ello concluimos, una gran ampliación de éstas últimas, fruto de la incorporación de España a la esfera internacional y en segundo lugar, la configuración de una naturaleza administrativa con connotaciones institucionales. 
Keywords: Administration, legal regulations, missions, institutional.

Palabras clave: Administración, régimen jurídico, misiones, institucional.

Recepción original: 10/06/2013

Aceptación original: 11/06/2013

Sumario: 1. Presentación 2. Historia reciente de las Fuerzas Armadas 3. Naturaleza de las Fuerzas Armadas 4. Composición 5. Estructura 6. Misiones constitucionales de las Fuerzas Armadas

\section{PRESENTACIÓN}

El Gobierno, según el artículo 97.1 de la Constitución, dirige la administración civil y militar del Estado.

Pues bien, es en su artículo 8 donde la Constitución española define a las Fuerzas Armadas: «... constituidas por el Ejército de Tierra, la Armada y el Ejército del Aire, tienen como misión garantizar la soberanía e independencia de España, defender su integridad territorial y el ordenamiento constitucional». Según el artículo 149.1.4. ${ }^{a}$ de la Constitución, asímismo, la competencia en materia de Defensa y Fuerzas Armadas corresponde al Estado.

Bien, pues precedentes de tal constitucionalización los encontramos en el Título VIII de la Constitución de Cádiz de 1812, en la que se crearon tropas de continuo servicio y milicias nacionales. Dice el artículo 356 de tal Constitución: «Habrá una fuerza militar nacional permanente, de tierra y de mar, para la defensa exterior del Estado y la conservación del orden interior». Y en el artículo 362, inscrito en el Capítulo II (De las milicias nacionales), del Título VIII, se dice «Habrá en cada provincia cuerpos de milicias nacionales, compuestos de habitantes de cada una de ellas, con proporción a su población y circunstancias». Aclara el artículo 364 que el servicio de estas milicias no sería continuo y sólo tendría lugar cuando las circunstancias lo requiriesen. Además, el Rey podría disponer de esta fuerza dentro de la respectiva provincia pero no podría emplearla fuera de ella sin la autorización de las Cortes.

Más reciente y premonitoriamente en el artículo 37 de la Ley Orgánica del Estado del régimen franquista:

«Las Fuerzas Armadas de la Nación, constituidas por los Ejércitos de Tierra, Mar y Aire y las Fuerzas de Orden Público, garantizan 
la unidad e independencia de la Patria, la integridad de sus territorios, la seguridad nacional y la defensa del orden institucional».

Y en derecho comparado también cabe hallar artículos similares. Así el artículo 87 de la Ley fundamental de Bonn de 1949. Dice su apartado a:

«1. La Federación organizará las Fuerzas Armadas para la defensa. Los efectivos numéricos y las características fundamentales de la organización deberán expresarse en el presupuesto.

2. Fuera de la defensa, las Fuerzas Armadas podrán utilizarse sólo cuando así lo autorice expresamente la presente Ley Fundamental.

3. En los casos de defensa y de tensión, las Fuerzas Armadas estarán autorizadas para proteger objetos civiles y asumir tareas de regulación del tráfico, en tanto que ello sea necesario para la realización de su misión de defensa. Además, en los casos de defensa y de tensión, podrá también confiarse a las Fuerzas Armadas la protección de objetos civiles para apoyar así medidas policiales; en estos casos, las Fuerzas Armadas cooperarán con las autoridades competentes.

4. Para prevenir un peligro inminente para la existencia o el régimen fundamental de libertad y democracia de la Federación o de un Land, el Gobierno Federal podrá, siempre que se den los requisitos del artículo 91, apartado 2, y cuando no resultaren suficientes las fuerzas de la policía y del Cuerpo Federal de Protección de las Fronteras, utilizar las Fuerzas Armadas para apoyar a la policía y al Cuerpo Federal de Protección de las Fronteras en la protección de objetos civiles y para luchar contra sediciosos organizados y militarmente armados. Deberá cesar la actuación de las Fuerzas Armadas cuando así lo exigieran el Bundestag o el Bundesrat.»

Vemos, pues, cómo la Ley Fundamental de Bonn extrema la precisión en su redacción y contempla, además, misiones no propiamente militares y que en España hemos tardado décadas en incorporar a nuestro acervo legislativo, como veremos más adelante, como la cooperación con la Policía en la realización de tareas más propiamente policiales.

Citamos, por último, el artículo 79 de la Constitución austríaca de 1920, que hace corresponder al Ejército federal la defensa nacional del país, pero, además en la medida en que la potestad civil 
lo exija, tendrá como misiones: «1) Las siguientes tareas fuera del ámbito de la defensa militar del país: a) la protección de las instituciones constitucionales y de su capacidad de funcionamiento, así como de las libertades democráticas de los habitantes, b) al mantenimiento del orden y la seguridad interiores. 2) Prestar ayuda en caso de sucesos y accidentes de magnitud extraordinaria».

La Constitución austríaca en el apartado 5 de tal artículo 79 añade que: «Sólo se permitirá la intervención militar independiente con los fines especificados en el párrafo 2 cuando las autoridades competentes no se encuentren, por causa de fuerza mayor, en condiciones de promover dicha intervención y sea previsible algún daño irreparable para la colectividad como consecuencia de una mayor dilación, o bien cuando se trate de eliminar una resistencia violenta (gewalttatiger Widerstand) o de rechazar un ataque efectivo dirigido contra algún sector del Ejército federal.»

Una intervención militar independiente de la que podríamos decir que está muy lejos de ser admitida en nuestra doctrina constitucional, hoy, si queremos ser respetuosos con el principio de supremacía civil, uno de los que regulan las Fuerzas Armadas.

Finalmente, el artículo 273.1 de la Constitución portuguesa de 1976, dice que las «Fuerzas Armadas portuguesas garantizarán la independencia nacional, la unidad del Estado y la integridad del territorio». Añadiendo el apartado 3 que «Las Fuerzas Armadas portuguesas garantizarán el funcionamiento regular de las instituciones democráticas y el cumplimiento de la Constitución».

En ella, como han afirmado los propios ponentes de la Constitución española de 1978, cabe rastrear algunas influencias, a pesar del marcado sello revolucionario posterior al movimiento del 25 de abril de 1974.

En el ámbito infraconstitucional español, cabe mencionar varias leyes que han ido conformando con el paso del tiempo el marco jurídico en el que se inscriben los militares. Mencionaremos ahora, entre otras, la Ley 5/2005 de 17 de noviembre de la Defensa Nacional, primera ley del Gobierno Zapatero que, como dice su artículo 1, regula la defensa y establece las bases de la organización militar conforme a lo establecido por la Constitución. Entre otras cosas: a) Actualiza el modelo organizativo de la defensa nacional b) Incluye misiones de las Fuerzas Armadas no recogidas en la anterior normativa c) Crea una nueva modalidad de participación de las Cortes Generales en el control de las decisiones gubernamentales que im- 
pulsen nuevas misiones en el exterior d)Redistribuye competencias en materia de Defensa Nacional entre el Presidente del Gobierno, Ministro de Defensa y Cortes Generales e) Crea el Consejo de Defensa Nacional f) Implanta una nueva organización en la que se diferencia el mando orgánico y el operativo g) Subraya los principios de jerarquía, disciplina, unidad y eficacia h) Regula las reglas esenciales de comportamiento de los militares i) Establece el principio de contribución gradual y proporcionada a la Defensa Nacional j) Por último, crea la figura del reservista.

\section{HISTORIA RECIENTE DE LA DEFENSA NACIONAL}

El 14 de noviembre de 1996, el Congreso aprobó la autorización al Gobierno de José María Aznar para que negociase el ingreso de España en la Nueva Estructura de Mandos de la OTAN. De este modo, en 1997, el Comité Militar de la OTAN, acordó en Maastricht crear el Mando Conjunto Subregional del Sudoeste, subordinado al Mando Regional Sur e integrado en el Mando Estratégico de Europa.

El 1 de enero de 1999, España entra en la estructura militar de la OTAN y seis meses después se constituye el Cuartel General del nuevo mando, con sede en Madrid, con competencias operativas sobre las unidades desplegadas en todo el territorio español, incluyendo Canarias, una región, como se sabe, de gran interés estratégico para España.

En otro orden de cosas y retrocediendo un poco en el tiempo, en 1994, el aún Gobierno socialista había decidido aportar tropas al Cuerpo de Ejército Europeo.

En efecto, el Eurocuerpo había sido constituido por los presidentes Miterrand y Kohl el 22 de mayo de 1992 en La Rochelle. Bélgica se sumó a esta iniciativa un año después y el 1 de julio de 1994, España aportó una División mecanizada. La integración definitiva se produjo el 31 de diciembre de 1998 con la incorporación de la Brigada de Infantería Mecanizada Extremadura XI y las unidades restantes del Núcleo de Tropas Divisionario (NTD) de la División Mecanizada Brunete.

La decisión de emplear esta unidad es competencia de cada Gobierno. Actualmente, los gobiernos podrían emplear el Eurocuerpo en el marco de la Unión Europea, en el marco de la OTAN o bien de forma independiente. Sus misiones son: a) Defensa común de los aliados en aplicación de los artículos 5 y V de los Tratados de Wash- 
ington y Bruselas, respectivamente b) Mantenimiento y restablecimiento de la paz c) Acciones humanitarias.

Simultáneamente a todo este proceso que hemos narrado, la desaparición del Pacto de Varsovia en 1989 había cuestionado la virtualidad de la OTAN. Sin embargo, el aumento de la participación de tropas de la OTAN y de la UEO en Operaciones de Mantenimiento de la Paz permitió a los Gobiernos occidentales justificar su existencia. Desde entonces, sus objetivos fueron la defensa del orden occidental y los derechos humanos de los pueblos.

Desde el primer momento, el presidente González alentó a su ministro de Asuntos Exteriores, Francisco Fernández Ordóñez, a que impulsara la participación militar de España en dichas misiones. La primera misión fue la UNAVEM I, Misión de verificación de las Naciones Unidas en Angola, en 1989. A ella le siguieron otras muchas en Namibia (UNTAG), Nicaragua (ONUCA), Haití (UNAVEH), Irak (Provide Comfort), El Salvador (ONUSAL), Macedonia(ECTF), Ruanda (UNAMIR), Mozambique (ONUMOZ), o Bosnia, habitualmente cumpliendo los mandatos de Naciones Unidas.

En efecto, son destacables las misiones llevadas a cabo en Bosnia o en Kosovo. La primera se inició primero bajo los auspicios de Naciones Unidas (UNPROFOR). Después, tras los acuerdos de Dayton, continuó bajo la cobertura de la OTAN, como Fuerza de Implementación (IFOR). Y finalmente, como Fuerza de Estabilización (SFOR). En el caso de Kosovo, la presencia española comienza en 1998, bajo el paraguas de la OSCE y continuó bajo mandato de la OTAN como Fuerza de Seguridad para Kosovo (KFOR), en la que modestamente, tuvimos la oportunidad de participar en el año 2007 con la agrupación llamada KSPFOR XVII.

Los atentados «yihadistas» contra las Torres Gemelas y el Pentágono del 11 de septiembre de 2001 influyeron de forma capital en la Revisión Estratégica de la Defensa, documento que pretendía definir a medio y largo plazo el modelo de ejército para el siglo XXI y que fue consensuado por todas las fuerzas políticas del arco parlamentario; documento que reproduciremos más adelante.

Ya en enero de 2002, siguiendo con nuestro relato, se incorporó a la Fuerza Internacional de Asistencia y Seguridad para Afganistán (ISAF), un contingente de tropas españolas y con ello, ya se elevaron a 3000 los españoles desplegados fuera de nuestras fronteras.

En abril de 2003, una vez acabada la guerra de Irak, una Unidad Conjunta de Apoyo Humanitario se trasladó a la ciudad portuaria 
de Um Qasr para colaborar en labores de reconstrucción del país. Y poco después, España envió otros 1300 efectivos para integrase en la brigada hispanoamericana desplegada en Nayaf y Diwaniyah con la misión de estabilizar la región y prestar apoyo humanitario.

El Gobierno socialista de José Luis Rodríguez Zapatero, en una decisión, a nuestro juicio imprudente, ordenó, nada más llegar a la presidencia del Gobierno el repliegue de las tropas españolas. En compensación y suponemos que desde la mala conciencia de abandonar a un aliado en mitad de la pelea, se acordó intensificar la ayuda a la reconstrucción de Afganistán: se constituyó un Equipo de Reconstrucción Provincial en Qala i Naw y una base logística junto con su hospital de campaña en Herat, al sur.

Actualmente, las Fuerzas Armadas se encuentran en misión en Afganistán, Líbano y Somalia.

Todas estos despliegues en zona de operaciones, que constituyeron la primera vez en doscientos años que el ejército recuperó su proyección internacional de manera permanente tuvieron, han tenido y tienen (en la actualidad, hay presencia española en Afganistán, Líbano, Somalia) muy benéficas consecuencias en los cuadros de mando y en la tropa: revitalizó las estructuras castrenses, modernizó u obligó a modernizar los ejércitos desde el punto de vista humano y material, motivó a los soldados, hizo que los oficiales volvieran de nuevo su vista al entorno internacional, como antaño hicieron sus predecesores, etc.

\section{NATURALEZA DE LAS FUERZAS ARMADAS}

El conocimiento de la naturaleza de las Fuerzas Armadas nos lleva inevitablemente a aclarar si éstas son institución o administración, extremo éste que ha sido objeto de numerosos debates doctrinales. Quizás, dado que en las Fuerzas Armadas se observan notas propias de una institución y de una administración, la actitud más acertada sea la capaz de acoger postulados de una y otra posición doctrinal.

Como es sabido, la Administración es el aparato organizativo que, bajo la dirección del Gobierno, desarrolla las funciones y realiza las tareas del Estado que no corresponden a otros poderes constitucionales y que está regida por un Derecho peculiar y propio, el Administrativo, cuyo principio inspirador es el principio de legalidad. 
Por el contrario, la idea de institución, desde el punto de vista sociológico, hace referencia a la idea de obra o empresa, un poder y una comunión entre los miembros para llevar a cabo tal idea (Hauriou) ${ }^{1}$ o bien, en palabras de Georges Renard, se diría que la institución aparece cuando la comunión en una idea o fin agrupa a los individuos y da nacimiento a un cuerpo $^{2}$; y desde el punto de vista jurídico, es un haz de relaciones jerárquicamente estructuradas en función de una meta que las trasciende.

Pues bien, en general, la doctrina jurídica actual es partidaria de caracterizar a las Fuerzas Armadas como administración y no como institución, si bien éste último término no deja de emplearse constantemente en el lenguaje corriente e incluso por los mismos autores que afirman el carácter administrativo de las Fuerzas Armadas.

En España, defienden la concepción administrativa autores como José Jiménez Villarejo ( «la situación de los militares no se diferencia demasiado de la del resto de los funcionarios de la Administración Pública...»³), Fernando López Ramón («no parece posible, en efecto, reducir el significado de la Administración militar a las estructuras ministeriales de la Administración del Estado, es decir, en la actualidad, al Ministerio de Defensa»", lo que, "sensu contrario», quiere decir que la Administración militar se extiende hasta las unidades militares) o Gustavo Suárez Pertierra (afirma «la progresiva administrativización de las Fuerzas Armadas $»^{5}$ y niega que se cumplan en las Fuerzas Armadas los caracteres fundamentales de la idea de institución: autonomía de organización, de disposición de medios y de decisión sobre los fines).

En efecto, parece que la posición administrativista es la acogida en el artículo 97 de la Constitución española de 1978, a juzgar por su tenor literal: «El Gobierno dirige la política interior y exterior, la admi-

1 Hauriou, M., La théorie de l'institution et de la fondation, Cahiers de la Nouvelle Journée, n. ${ }^{\circ}$ 4, París, 1925, págs. 2-45.

2 Renard, G., La Théorie de l'institution, Paris, Sirey, 1930, pág. 95.

3 Jiménez Villarejo, J., Derechos y deberes del militar profesional en la Constitución española, en Revista Española de Derecho Militar n. ${ }^{\circ}$ 77, Madrid, Ministerio de Defensa (Secretaría General Técnica), 2001, pág. 448.

4 López Ramón, F., Principios de Ordenación Constitucional de las Fuerzas Armadas, en Estudios sobre la Constitución española. Homenaje al profesor Eduardo García de Enterría. Tomo III. La Corona. Las Cortes. Del Gobierno y la Administración Pública, Madrid, Civitas, 1991, pág. 2568.

5 SuÁrez Pertierra, G., Regulación jurídico constitucional de las Fuerzas Armadas, en Jornadas de Estudio sobre el Título Preliminar de la Constitución, Volumen IV, Madrid, Ministerio de Defensa, Secretaría General Técnica, Centro de Publicaciones, 1988, pág. 2375. 
nistración civil y militar y la defensa del Estado...». Por tanto, se diría que las Fuerzas Armadas son administración y que la administración militar es parte de la administración del Estado y se compone de la Fuerza, propiamente dicha (término militar que denomina al conjunto de las unidades militares que tienen como misión la más directa ejecución de las operaciones) y de toda una estructura funcionarial que sirve a los propósitos de la Fuerza: el Apoyo a la Fuerza (término militar que designa, como hemos apuntado a todas las unidades que brindan apoyo logístico, sanitario, etc a las unidades operativas). Además, estas Fuerzas Armadas, en cuanto administración del Estado, estarían (y están) igualmente sometidas a los principios de legalidad (dice el artículo 103. 1 de la Constitución que «La Administración Pública sirve...con sometimiento pleno a la ley y al Derecho») y al principio de dependencia del poder civil (art. 97.1 de la Constitución, antes citado). El Tribunal Constitucional, a mayor abundamiento en este argumento, en su sentencia 15/1982, de 23 de abril, se refiere explícitamente a la «Administración militar» y no a la institución militar.

En la Ley 6/1980 de 1 de julio por la que se regulaban los criterios básicos de la defensa nacional y la organización militar, es patente la desaparición del poder militar autónomo (una de las notas de una institución) y queda suficientemente garantizada la subordinación de las Fuerzas Armadas al poder civil. Dice el artículo 8 de dicha ley: «El Presidente del Gobierno dirige y coordina la acción del mismo en materia de defensa. Corresponde, asimismo, al Presidente del Gobierno ejercer la dirección de la guerra, asistido por la Junta de Defensa Nacional.»

Posteriormente, la Ley 1/1984, de 5 de enero, de reforma de la Ley Orgánica 6/1980 de 1 de julio, por la que se regulan los criterios básicos de la Defensa Nacional y la Organización Militar, como explicaremos en un epígrafe posterior, aclaró y confirmó esta subordinación, lo que podríamos decir que acentuó aún más en este carácter administrativo.

La ley 5/2005 de 17 de noviembre de la Defensa Nacional, por fin, declara en su artículo 5 que «Corresponde al Gobierno determinar la política de defensa y asegurar su ejecución, así como dirigir la Administración militar y acordar la participación de las Fuerzas Armadas en misiones fuera del territorio nacional». Y en su artículo 6 dice: «Corresponde al Presidente del Gobierno la dirección de la política de defensa y la determinación de sus objetivos, la gestión de las situaciones de crisis que afecten a la defensa y la dirección estratégica de las operaciones militares en caso de uso de la fuerza». 
Por otra parte, son numerosas las normas administrativas que se han extrapolado al ámbito militar: normas referidas a jornada de trabajo, horarios, vacaciones, permisos, licencias; normas que hasta ahora podíamos encontrar en el Estatuto Básico del Empleado Público y ahora se han extendido a la función pública militar con el fin de homologar ambos «status quo». Lo que da cuenta de que para el legislador ordinario y orgánico, no deben existir diferencias normativas entre la administración civil y las Fuerzas Armadas, sobre todo en materia de régimen de personal.

En el otro extremo, se encuentran autores como Federico Trillo Figueroa (dice que éstas son una «unidad institucional»), Nevado Moreno (la posición institucional se puede apoyar en «la propia referencia constitucional de las Fuerzas Armadas al ubicarlas en el Título Preliminar, artículo 8, por lo que sería indudablemente una institución consagrada en el Texto Fundamental, auténtica categoría del Derecho») o Jorge de Esteban (en efecto, no se puede ignorar, de acuerdo con el tenor literal del artículo 8.1 de la Constitución, dice Jorge de Esteban, «la significación institucional» de que gozan las Fuerzas Armadas en nuestro ordenamiento constitucional, algo que se pone de manifiesto, por ejemplo, en el hecho de que figuren en el Título Preliminar, en plano de igualdad, junto a otras instituciones como los partidos políticos).

Ambas posiciones son solventes y sólidas. Pero como magistralmente explica Herrero de Miñón, «la propia Administración del Estado es una institución, o mejor, una institución de instituciones ${ }^{6}$. No vemos, por tanto, inconveniente en considerar a las Fuerzas Armadas como una administración de procedencia institucional o con connotaciones institucionales, pues aun siendo parte de la Administración del Estado, es evidente que está constituida por un grupo social con un estilo de vida propio, un código de valores particular, una organización propia, un ordenamiento jurídico, unas funciones distintas y un régimen jurídico y una cadena de mando jerarquizada propios.

\section{COMPOSICIÓN}

Las Fuerzas Armadas, de acuerdo con el artículo 8 de la Constitución, están compuestas por el Ejército de Tierra, la Armada y el Ejército del Aire. Por otra parte, dice el artículo 10 de la Ley 5/2005 de 17 de noviembre que: "Las Fuerzas Armadas son el elemento

6 Herrero de Miñón, M., El valor de la Constitución, Barcelona, Crítica, 2003, pág. 155. 
esencial de la defensa y constituyen una entidad única que se concibe como un conjunto integrador de las formas de acción específicas de cada uno de sus componentes: el Ejército de Tierra, la Armada y el Ejército del Aire».

Los miembros de las Fuerzas Armadas se integran o adscriben a distintos cuerpos, de acuerdo con los cometidos que deben llevar a cabo. Estos cuerpos pueden ser específicos de cada Ejército o comunes de las Fuerzas Armadas. Por ejemplo, infantería, artillería, ingenieros y caballería son parte de lo que se denomina el Cuerpo General de las Armas. Por el contrario, el Cuerpo Jurídico Militar es un cuerpo común, al igual que el de Sanidad Militar o Intervención, por mencionar algunos.

De otro lado, las Fuerzas Armadas se organizan, de acuerdo con el artículo 11 de la Ley 5/2005 en dos estructuras: una orgánica, para la preparación de la fuerza (batallones, regimientos, brigadas, divisiones, etc) y otra operativa, para su empleo en las misiones que se le asignen (por ejemplo, en el curso de una operación internacional de mantenimiento de la paz).

La estructura orgánica posibilita la generación de la estructura operativa. Se establece mediante criterios funcionales y de forma homogénea.

La estructura operativa, pensada para llevar a cabo acciones conjuntas y combinadas, se organiza con arreglo al principio de unidad de mando y a los criterios necesarios para conseguir la máxima capacidad operativa.

La composición de las Fuerzas Armadas no ofrece muchas discusiones doctrinales. Más interés ofrece discernir quiénes no forman parte de las Fuerzas Armadas. A ello dedicamos las siguientes líneas.

En primer lugar, las Fuerzas y Cuerpos de Seguridad del Estado no forman parte de las Fuerzas Armadas pues «su naturaleza, su reconocimiento constitucional y sus funciones son muy diferentes» ${ }^{7}$ : a) En efecto, las Fuerzas y Cuerpos de Seguridad son civiles y no militares b) Además, el reconocimiento del que gozan ambas instituciones por parte de la Constitución es muy distinto, pues el artículo 8.1 habla únicamente de las Fuerzas Armadas, mientras el artículo 104 trata de las Fuerzas y Cuerpos de Seguridad c) Por último sus misiones son distintas: las Fuerzas Armadas tienen como cometido la

7 De Esteban, J., GonzÁlez Trevijano, P., Tratado de Derecho Constitucional III, Colección Textos, Madrid, Servicio de Publicaciones de la Facultad de Derecho, 1994, pág. 358. 
defensa exterior, pues como reza el artículo 8, garantizan la soberanía e independencia de la patria; mientras las Fuerzas y Cuerpos de Seguridad del Estado lo hacen en el interior, pues protegen el libre ejercicio de los derechos y libertades fundamentales y garantizan la seguridad ciudadana.

No obstante, no ignoremos que las Fuerzas y Cuerpos de Seguridad del Estado también pueden contribuir a la Defensa Nacional, bajo la dependencia de la autoridad militar en caso de declaración de estado de sitio, de acuerdo con la Ley Orgánica 4/1981 de 1 de junio, de los estados de alarma, excepción y sitio y de acuerdo con el artículo 27 de la Ley 5/2005 de 17 de noviembre de la Defensa Nacional, según el cuál, el Cuerpo Nacional de Policía, en caso de conflicto bélico, será coordinado por el Consejo de Defensa Nacional, aun dependiendo del Ministro del Interior.

Por su parte, la Guardia Civil, que «tradicionalmente ha venido formando parte del Ejército de Tierra ${ }^{8}$, desde 1980 y hasta hoy, de acuerdo con el artículo 23 de la Ley 5/2005 de 17 de noviembre de la Defensa Nacional, «es un instituto armado de naturaleza militar, dependiente, del Ministerio de Interior en el cumplimiento de misiones que le atribuye la Ley Orgánica de Fuerzas y Cuerpos de Seguridad 2/1986 de 13 de marzo y del Ministerio de Defensa en el cumplimiento de las misiones de carácter militar que se le encomienden...»

\section{ESTRUCTURA}

A continuación, nos disponemos a resumir el Real decreto 912/2002 por el que se desarrolla la estructura básica de los Ejércitos:

En primer lugar, como el propio decreto reza, se trata de implantar una estructura conjunta, no redundante y guiada por el principio de economía de medios. Respecto a su estructura, dice que los ejércitos, bajo el mando de sus respectivos jefes de estado mayor, se organizan en Cuartel General, Fuerza y Apoyo a la Fuerza.

El Cuartel General está constituido por los medios humanos y materiales necesarios para que el Jefe del Estado Mayor pueda ejercer el mando sobre su Ejército.

La Fuerza se constituye por los medios humanos y materiales que se organizan y agrupan para llevar a cabo operaciones militares. En

8 Casado Burbano, P., Iniciación al Derecho Constitucional Militar, Madrid, Editoriales de Derecho Reunidas, 1986, pág. 26. 
este sentido, los Jefes de Estado Mayor, dice el Real decreto, deben mantener una organización y un despliegue de fuerzas que garantice que éstas se pueden asignar total o parcialmente al Jefe del Estado Mayor de la Defensa. La Fuerza principal del Ejército de Tierra se constituye de: Fuerza de Maniobra, Fuerza Terrestre y Fuerza Logística Operativa.

El Apoyo a la Fuerza es el conjunto de órganos responsables de la dirección, gestión, administración y control de los recursos materiales, financieros y humanos.

Se prevén, asimismo, Mandos o Jefaturas del Apoyo a la Fuerza. En el ámbito de los recursos humanos, serán los responsables de la dirección, gestión, administración y control, en materia de personal y en materia de sanidad y enseñanza. En el ámbito del apoyo logístico y de los recursos materiales, serán los principales responsables de la dirección, gestión, administración y control de material y del apoyo logístico y atenderán a la adquisición, abastecimiento, mantenimiento, transporte, infraestructura, sistemas de información y telecomunicaciones, los sistemas de armas y las construcciones, así como del apoyo en bases y acuartelamientos. Se prevén dos Mandos en el ámbito del apoyo logístico y de los recursos materiales: el Mando de Apoyo Logístico y el Inspector General del Ejército de Tierra.

Finalmente en Canarias y Baleares se mantienen las estructuras de mando preexistentes: el Mando de Canarias y las Comandancias Generales de Baleares, Ceuta y Melilla del Ejército de Tierra; el Mando Naval de Canarias de la Armada; el Mando Aéreo de Canarias del Ejército del Aire.

Se habilita, asimismo, al Gobierno a crear mandos conjuntos en los archipiélagos balear y canario.

Los Comandantes Generales de Ceuta y Melilla tendrán la consideración de mandos conjuntos y de ellos dependerán operativamente los mandos y unidades de los tres Ejércitos, con base en dichas ciudades. La dependencia operativa de ambas Comandancias será del Jefe del Estado Mayor de la Defensa.

Posteriormente y aún bajo el Gobierno del Partido Popular se procedió a una revisión de la Defensa y fruto de ello fue la Revisión Estratégica de la Defensa, un documento que pretendía plasmar las directrices que debían seguir las Fuerzas Armadas del Siglo XXI:

- «Encuadradas en el Ministerio de Defensa, las Fuerzas Armadas, elemento esencial de la Defensa, constituyen una entidad única que se concibe como un conjunto integrador de las 
formas de acción de cada uno de sus componentes: el Ejército de Tierra, la Armada y el Ejército del Aire. El Ministro de Defensa dirige su actuación, bajo la autoridad del Presidente del Gobierno, en el marco de la política de Defensa y con el control del Parlamento.

- El esfuerzo de Defensa debe estar orientado hacia la Fuerza, razón de ser de toda organización.

- Todas las unidades deberán estar preparadas para las acciones más exigentes del combate independientemente de que puedan desempeñar otro tipo de misiones.

- Las Fuerzas Armadas deberán estar configuradas para responder eficazmente a las nuevas misiones que se le encomienden en el marco conjunto y combinado.

- Al JEMAD, Comandante Operativo de las Fuerzas Armadas, le corresponderá la conducción estratégica de las operaciones y ejercerá el mando sobre los componentes de la estructura operativa de las Fuerzas Armadas. Se creará un Mando de Operaciones que, directamente subordinado al JEMAD ejercerá por delegación de éste, la conducción operacional.

- Se contará con una Fuerza Conjunta de Reacción Rápida, establecida sobre la base de un pool de fuerzas.

- A los Jefes de Estado Mayor les corresponderá el Mando Orgánico de su Ejército, sin ejercer el Mando Operativo.

- Se establecerá una estructura funcional para los Ejércitos.

- El recurso humano constituye el elemento fundamental de la Defensa. En consecuencia, se llevarán a cabo las acciones necesarias para conseguir un personal suficiente, altamente motivado y preparado.

- Se potenciará la máxima integración logística de la Defensa.

- Se avanzará hacia la máxima interoperabilidad con los aliados y socios europeos.

- Las Fuerzas Terrestres deberán tener la mayor capacidad de proyección y despliegue, movilidad táctica y capacidad resolutiva.

- Las Fuerzas Navales, manteniendo su capacidad de control de los espacios marítimos de soberanía e interés nacional, deben estar orientadas hacia las operaciones en teatros litorales leja- 
nos, con especial énfasis en proyección del poder naval sobre tierra.

- Las Fuerzas Aéreas han de tener capacidad de combate en todo tiempo, mayor capacidad de transporte y mayor alcance y precisión» ${ }^{9}$.

En 2004, el Gobierno de José Luis Rodríguez Zapatero llevó a cabo una nueva reforma recogiendo la aportación del anterior documento y concibiendo a las Fuerzas Armadas dentro de esa dualidad que podemos apreciar en él: a) Se creó el Mando de Operaciones, subordinado al Jefe de Estado Mayor de la Defensa, que tendría como misión, a partir de ese momento, la conducción de las operaciones en tiempo de paz, crisis, guerra, conflicto armado, así como el control de los servicios de inteligencia militar b) Desde el punto de vista orgánico, cada ejército siguió dependiendo del respectivo Jefe de Estado Mayor, responsable de garantizar la asignación de fuerzas al citado Mando Operativo.

\section{MISIONES CONSTITUCIONALES DE LAS FUERZAS ARMADAS}

En este extremo, el de saber qué misiones tienen encomendadas las Fuerzas Armadas, debemos seguir el tenor del texto constitucional y el de la reciente Ley 5/2005 de 17 de noviembre de la Defensa Nacional.

En suma cabría hablar de seis misiones, tres de ellas declaradas por la Constitución (art.8.1) y tres declaradas por la Ley 5/2005 de 17 de noviembre en su artículo 15. Son: a) Garantizar la soberanía e independencia de la patria b) Defender la integridad territorial c)Defender el ordenamiento constitucional d) Participar en operaciones de mantenimiento de la paz, estabilidad y de ayuda humanitaria e) Intervenir en caso de riesgo, catástrofe o calamidad pública f) Misiones de evacuación de españoles en el extranjero.

El artículo 8.1 de la Constitución declara, en primer lugar, que las Fuerzas Armadas deben garantizar la soberanía e independencia de la patria, defender la integridad territorial y el ordenamiento constitucional. El primer precedente de este texto lo encontramos en la ya mencionada Constitución de 1812, que encomendaba a la Fuerza

9 Ministerio de Defensa, Revisión Estratégica de la Defensa. I. Planteamiento General. II. Criterios Básicos, Revista Española de Defensa, Suplemento del N. 180 , Madrid, Ministerio de Defensa (Secretaría General Técnica) 2003. 
Militar Permanente «la defensa exterior del Estado y la defensa del orden interior» (art. 356). Posteriormente, encontramos precedentes en leyes como la Ley Constitutiva del Ejército, de 9 de junio de 1821, que hablaba de «defender el Estado de los enemigos exteriores» y «asegurar la libertad política, el orden público y la ejecución de las leyes». También la Ley constitutiva de 29 de noviembre de 1878, que señalaba como misión del Ejército «sostener la independencia de la Patria y defenderla de enemigos exteriores e interiores». Asímismo, la Ley adicional de 19 de julio de 1889, consignaba como misión del Ejército «mantener la independencia e integridad de la Patria y el imperio de la Constitución y de las leyes». Por último, la Ley Orgánica del Estado del anterior régimen franquista, en su artículo 37, presenta una redacción muy semejante a la de la actual Constitución: «Las Fuerzas Armadas de la Nación, constituidas por los Ejércitos de Tierra, Mar y Aire y las Fuerzas de Orden Público, garantizan la unidad e independencia de la Patria, la integridad de sus territorios, la seguridad nacional y la defensa del orden institucional».

Primera: Garantizar la soberanía e independencia de la Patria

Es la misión clásica de «defender con las armas a la comunidad frente a las agresiones externas ${ }^{10}$, en palabras del profesor Casado Burbano. Dicho de otro modo se trata de una misión vinculada «a la residencia de la soberanía en el pueblo español y a la independencia o autonomía de decisión del pueblo español en el orden internacional» ${ }^{11}$.

No obstante, la fuerza siempre será la última ratio y por tanto, las Fuerzas Armadas se deben emplear como disuasión y de acuerdo con un principio defensivo. De hecho, el Preámbulo de la Constitución de 1978 dice que es misión de la Nación española «colaborar en el fortalecimiento de unas relaciones pacíficas y de eficaz cooperación entre todos los pueblos de la Tierra».

Ello no impide, empero, que España se incorpore a organizaciones internacionales como la Unión Europea, la Unión Europea Occidental o la Organización del Tratado del Atlántico Norte. Orga-

10 Casado Burbano, P., Las Fuerzas Armadas en la Constitución Española, en Revista Española de Derecho Militar, n. ${ }^{\circ}$ 36, Madrid, Instituto Francisco de Vitoria. Sección de Derecho Militar. CSIC, 1978, pág. 15.

11 De Juan González de Castejón, I., La Defensa Nacional: desarrollo del artículo 8 de la Constitución, en La Constitución y la práctica del Derecho, T. II., Pamplona, Aranzadi, 1998, pág. 580. 
nizaciones estas dos últimas, que como hemos dicho anteriormente, tras la caída del Pacto de Varsovia han redefinido su misión y han encontrado por tanto, un nuevo rol en el mundo: han pasado de ser una organización militar pensada para proteger a sus miembros de ataques procedentes de los países del este y de la Unión Soviética a ser organizaciones que velan por la protección de los derechos humanos de los pueblos y de sus ciudadanos allí donde sean vulnerados: Kosovo, Afganistán, etc

\section{Segunda: Defender la integridad constitucional}

Ésta misión tiene a su vez, dos dimensiones: a) Externa: impedir cualquier amago de violar nuestra integridad territorial desde fuera de nuestras fronteras b) Interna: impedir secesiones por parte de sujetos políticos internos (como ocurriría en el caso de una eventual declaración de independencia por parte de una región española). Esta dimensión se fundamenta en la declaración que hace el artículo 2 de la Constitución, según el cuál, ésta «se fundamenta en la indisoluble unidad de la Nación española».

Como información podemos mencionar, que en los debates parlamentarios que se sucedieron en el seno de las comisiones encargadas de redactar la futura Constitución de 1978, el senador D. Manuel Fraga Iribarne, de Alianza Popular, propuso integrar en el texto constitucional la idea de «la defensa de la unidad». Sin embargo, la mayoría de los ponentes constitucionales entendieron que tal idea quedaba ya incluida en la defensa del orden constitucional «por cuanto en la unidad indisoluble de la Nación española se fundamenta la propia Constitución, según su artículo $2 »^{12}$.

\section{Tercera: Defender el ordenamiento constitucional}

Ésta misión, citando a Oehling, es una misión habitual en las constituciones de "aquéllos países en cuya historia política ha sido frecuente la incursión del estamento armado, o en que se manifiesta el ánimo temeroso de tal posibilidad ${ }^{13}$. Siguiendo a López Ramón,

12 Suárez Pertierra, G., Regulación Jurídico Constitucional de las Fuerzas Armadas, en Jornadas de Estudio sobre el Título Preliminar de la Constitución, Volumen IV, Madrid, Ministerio de Defensa, Secretaría General Técnica, Centro de Publicaciones, 1988, pág. 2386.

13 Oehling, H, «La función política del Ejército», Instituto de Estudios Políticos, Madrid, 1967, pág. 70. 
esta misión está relacionada con la «defensa interior del Estado» y consistiría en la defensa del «conjunto formado por el sistema institucional y el normativo» ${ }^{14}$. Esta misión, en opinión del profesor Jorge de Esteban, está sometida a los siguientes parámetros: a) Es una misión que sólo procede en el caso de declaración del Estado de sitio (art. 116. 4), por lo que las Fuerzas Armadas, por sí mismas, no pueden llevarla a cabo. b) Es una función de carácter extraordinario y disuasorio. c) Y además, será bajo las directrices del poder civil como se ejercerá tal cometido pues, de acuerdo con el artículo 33.2 de la Ley Orgánica 4/1981, de 1 de junio, de los estados de alarma, excepción y sitio «el Gobierno designará la autoridad militar que deba ejercer las medidas que procedan». d) Es, por último, una defensa política, material, no jurídica pues para ello ya está el Tribunal Constitucional. ${ }^{15}$

Cuarta: Operaciones de mantenimiento de la paz, la estabilidad y la ayuda humanitaria

Dice el artículo 15.2 de la Ley 5/2005 de 17 de noviembre de la Defensa Nacional que «Las Fuerzas Armadas contribuyen militarmente a la seguridad y defensa de España y de sus aliados, en el marco de las organizaciones internacionales de las que forma parte así como al mantenimiento de la paz, la estabilidad y la ayuda humanitaria».

Se trata de misiones como aquéllas en las que, modestamente, hemos tenido la oportunidad de participar, como la llevada a cabo en Kosovo en 2007 (KSPGT XVIII), en Líbano, en 2008 (L-H VII) u otras en las que no hemos participado como la aún vigente en Afganistán, en las que se trata de mantener la libertad de movimientos y el ambiente seguro y estable, en las que la Fuerza dispone de unas R.O.E. o reglas de enfrentamiento y están amparadas por una resolución de Naciones Unidas o de la OTAN y de las que, desgraciadamente, la opinión pública no tiene el más mínimo conocimiento, lo que, a menudo impide, como decía Tocqueville al analizar el papel

14 López Ramón F., Principios de Ordenación Constitucional de las Fuerzas Armadas, en Estudio sobre la Constitución Española. Homenaje al profesor Eduardo García de Enterría. Tomo III. La Corona. Las Cortes. Del Gobierno y de la Administración Pública, Madrid, Civitas, 1991, pág. 2586.

15 De Esteban, J., González TreviJano, P., Tratado de Derecho Constitucional III, Colección Textos, Madrid, Servicio de Publicaciones de la Facultad de Derecho, 1994 (1. ' reimpresión 2000), pág. 360. 
de la prensa en la democracia estadounidense, «actuar libremente en la realidad $»^{16}$.

Quinta: Preservar la seguridad y el bienestar de los ciudadanos en los supuestos de grave riesgo, catástrofe, calamidad u otras necesidades públicas

Ésta es otra de las misiones que recientemente se han incorporado a las leyes y que las Fuerzas Armadas deben llevar a cabo junto con las Instituciones del Estado y las demás Administraciones Públicas. Baste decir que esta misión ha sido desempeñada por las Fuerzas Armadas, por ejemplo, con ocasión de la crisis de los controladores, en 2011, incendios forestales de grandes proporciones, atentados terroristas como los del 11 de marzo de 2004, que motivó que el Ejército patrullase a lo largo de las vías del ferrocarril, etc

Sexta: Llevar a cabo la evacuación de residentes españoles en el extranjero

Esta es una misión que deben llevar adelante las Fuerzas Armadas en el caso de que ocurran circunstancias de inestabilidad en un país de tal modo que pongan en grave riesgo su vida o sus intereses. Ejemplos de ésta última misión podemos rastrearlos en la evacuación de españoles de Líbano en el año 2005, la de españoles afincados en países africanos recientemente desestabilizados como el Congo, etc

A su vez, como recuerda el artículo 16 de la Ley 5/2005 de 17 de noviembre, el cumplimiento de las misiones de las Fuerzas Armadas y el desarrollo de su contribución complementaria o subsidiaria de interés público requieren realizar diferentes tipos de operaciones, tanto en territorio nacional como en el exterior, que pueden conducir a acciones de prevención de conflictos o disuasión, de mantenimiento de la paz, actuaciones en situaciones de crisis y, en su caso, de respuesta a la agresión. En particular, las operaciones pueden consistir en:

a) La vigilancia de los espacios marítimos, como contribución a la acción del Estado en la mar, la vigilancia del espacio aéreo y el control del espacio aéreo de soberanía nacional y aquellas otras actividades destinadas a garantizar la soberanía e independencia de España, así como a proteger la vida de su población y sus intereses.

16 Tocoueville, A., «La democracia en América», Madrid, Alianza Editorial, 2005, pág. 265. 
b) La colaboración en operaciones de mantenimiento de la paz y estabilización internacional en aquellas zonas donde se vean afectadas, reconstrucción de la seguridad y la administración, así como la rehabilitación de un país, región o zona determinada, conforme a los tratados y compromisos establecidos.

c) El apoyo a las Fuerzas y Cuerpos de Seguridad del Estado en la lucha contra el terrorismo y a las instituciones y organismos responsables de los servicios de rescate terrestre, marítimo y aéreo, en las tareas de búsqueda y salvamento.

d) La respuesta militar contra agresiones que se realicen utilizando aeronaves con fines terroristas que pongan en peligro la vida de la población y su interés. A estos efectos, el Gobierno designará la Autoridad nacional responsable y las Fuerzas Armadas establecerán los procedimientos operativos pertinentes.

e) La colaboración con las diferentes Administraciones públicas en los supuestos de grave riesgo, catástrofe, calamidad u otras necesidades públicas, conforme a lo establecido en la legislación vigente.

f) La participación con otros organismos nacionales e internacionales para preservar la seguridad y el bienestar de los ciudadanos españoles en el extranjero, de conformidad con los criterios de coordinación y de asignación de responsabilidades que se establezcan.

Respecto a estas operaciones, o mejor dicho respecto a la redacción que la Ley 5/2005 de 17 de noviembre, hace en su artículo 16 cabría observar lo siguiente:

En primer lugar, no deja de llamarnos la atención que un partido, como el socialista, que tanto se opuso al concepto de guerra preventiva cuando se encontraba en la oposición, una vez en el Gobierno consagra en la propia ley tal concepto cuando dice el artículo 16: «... acciones de prevención de conflictos...»

En segundo lugar, la letra d) del artículo 16 se introdujo, como se puede suponer, tras la situación creada por los ataques del 11 de septiembre de 2001 .

\section{CONCLUSIONES}

Las Fuerzas Armadas, como dijimos, son una administración con connotaciones institucionales. En efecto, pretender entender e interpretar las normas que rigen los Ejércitos al margen de los va- 
lores que dan sentido a este orden (un orden concreto como afirma Herrero de Miñón), haría que éstas perdieran todo sentido.

Nadie duda de que las Fuerzas Armadas están sometidas al principio de legalidad, ni que están subordinadas al Gobierno de la Nación, ni que el proceso de ingreso en las Fuerzas Armadas debe regirse por los mismos principios administrativos que en cualquier otra oposición, etc Pero, al mismo tiempo, no conocemos ninguna administración donde el principio de jerarquía esté tan acentuado, ni donde se arengue a los funcionarios por la mañana, con lectura de efemérides bélicas para fortalecer y transmitir valores morales, ni donde se haga jurar al funcionario a dar su vida por España, ni donde prime la disciplina y el cumplimiento de la misión sobre el debate, etc

Precisamente, calificar a las Fuerzas Armadas de orden concreto, es útil, siguiendo a Herrero de Miñón ${ }^{17}$, para aplicarle a las mismas una categoría germánica: la de garantía institucional, tal como la desarrolla el Tribunal Constitucional en su sentencia 32/1981 de 28 de julio, FJ 3..$^{\circ}$ «El orden jurídico-político establecido por la Constitución asegura la existencia de determinadas instituciones, a las que se considera como componentes esenciales y cuya preservación se juzga indispensable para asegurar los principios constitucionales, estableciendo en ellas un núcleo o reducto indispensable por el legislador... ${ }^{18}$

La garantía institucional de las Fuerzas Armadas explica su naturaleza última y más trascendental: el hecho de existir para garantizar la propia vida de los españoles y aun más, con el sacrificio de las propias vidas (de los militares). Sólo si atendemos a esta peculiar característica de las Fuerzas Armadas, entenderemos sus principios (como el de jerarquía o disciplina), su organización, su régimen jurídico, su modo de vida, etc.

17 Herrero de Miñón, M., El valor de la Constitución, Barcelona, Crítica, 2003, pág. 156.

18 STC 32/1981 de 28 de julio, FJ 3. ${ }^{\circ}$ 
